\title{
Evolution as pattern processing: TODO as substrate for evolution
}

\author{
P. Hogeweg \& B. Hesper \\ Bioinformatica Padualaan 8 , \\ $3584 \mathrm{CH}$ Utrecht the Netherlands \\ EMAIL: HESPER@RULCRI.Leiden.Univ.nl
}

\begin{abstract}
Evolution is most often viewed (and formalised) as an optimisation process. In this paper we suggest that a useful altemative heuristic may be to view (and formalise) evolution as a self-enhancing pattern transformation, pattem detection and pattern generation process. This suggestion is based on the growing awareness of the emergence of complex behaviour. from simple environmentally dependent action rules when they operate in a structured environment, and the structuring of the environment by such behavioural patterns. We describe paradigm worlds, which suggest that such emergent behaviour may underlie behaviour patterns observed in various species. We show how the emergence of macro-behaviour patterns can be interpreted as a form of pattern processing by the action rulcs on the environment. We suggest that these emergent patterns function as a prepattem for evolutionary processes: evolution fixates and enhances these patterns.
\end{abstract}

\section{Introduction}

Ever since Darwin's profound insight in equating 'survival' and 'fitness' for self-replicating entities, evolutionary thcory has had a strong footing in terms of optimisation processes. This optimisation viewpoint pervades most biological thinking about evolution and adaptation. Population genetics is entirely formulated in such terms, and traits of organisms are customarily 'explained' in tcrms of their 'fitness', recently in particular in sociobiology and behavioural ecology. Genetic Algorithms (Holland 1976, Goldberg 1989) have used basic 'genetic mechanisms' for solving general optimisation problems. Only relativcly recently have quantitative studics begun to expose the constraints on a 'mutation sclection' process leading to appreciable optimisation (after all: 'optimisation by 'survival of the fittest' is not a tautology). Eigen and Shuster (1979) exposed the 'error threshold', i.e. they showed that only a limited amount of mutation is compatible with evolutionary optimisation. Kauffman (Kauffman \& Smith 1986 Kauffman 1989a,b) stressed that optimisation is only possible in not 100 rugged fitness landscapes, i.e only if similar genotypes have, in general, similar fitnesses. Rugged fitness landscapes result from extensive coupling between genes, by which the system exhibits strong selforganising properties. Such selforganisation thus seems to be a constraint on evolutionary optimisation.

All these approaches use an external, apriori, 'user' imposed fitness criterion. Only a few models are studied, in which only survival determines the evolutionary process in a coevolutionary context. (Conrad \& Rizki 1989, Packard 1988, Holland 1990, Kauffman 1989b). In such models 'fitness' is not clearly defined, and fitness landscapes are wildly dynamic entities, if they can be visualised at all.

In this paper we propose that it may be worthwhile to view evolutionary processes not as primarily an optimisation process, but instead as pattern processing (i.e. as pattern detection, pattern transformation or pattern generation). Such a view is of course entirely compatible with the optimisation viewpoint, but provides different heuristics for studying such processes. In particular we should like to study what may be called the generation of 'fitness' dimensions, rather than walks through fixed fitness landscapes, although we prefer a terminology like 'pattern of survival' rather than fitness. The insight that pattern recognition and pattern detection can be studied in terms of encrgy minimisation ('optimisation' as used in evolutionary theory) (Hopficld 1984, Ackley et al. 1985 and the extensive literature which followed this conceptualisation) has led to important new models and machines for pattern recognition/ pattern detection. We hope that a similar, but reversed, change of viewpoint with respect to evolution (i.c. from optimisation to pattern detection) will likewise lead to new models elucidating (and possibly machines. exhibiting) innovative evolutionary adaptation.

\section{TODO and emergent behaviour}

\section{I Introduction to the TODO principle.}

The potential of local rules to generate complex behaviour in interaction with a structured environment was first hinted at by Simon (1969) in his phrase: "an Ant viewed as a behaving system is quite simple, the apparent complexity of its behaviour in time is largely a reflexion of the complexity of the environment in which it finds itself..."; "a Man viewed as a behaving system is quite simple, the apparent complexity of his behaviour in time is largely a reflexion of the complexity of the environment in which he finds himself...". Simon, and with him most Artificial Intelligence rescarch, have concentrated entirely on humans, and have in practice dismissed this phrase as an irony. Nor have those studying animal behaviour taken the hint seriously, they have continued to study behaviour virtually independent of the environment or they have paid attention to the environment as a constraint on (optimising) behaviour only. By contrast our own research has been in the direction of Simon's pointer, but has gone beyond it by

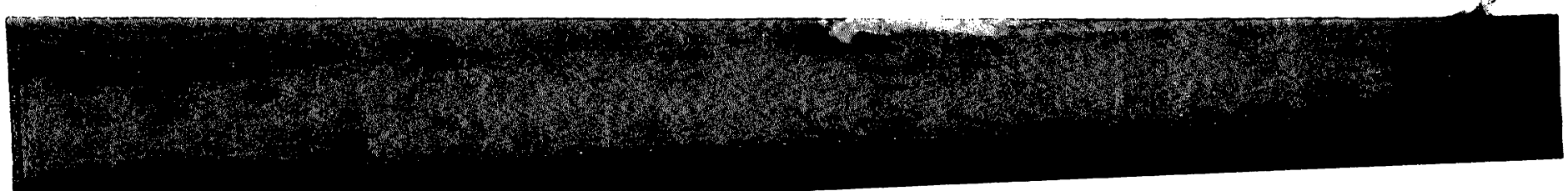


studying emergent self-regulating behaviour generated by entities governed by local rules in apriori lowly structured environments. We call the basic mechanism underlying this self-structuring the TODO principle: the entities 'do what there is to do'; what there is to do changes by their doings. In the following subsections we briefly summarise some insights which emerged from studying worlds governed by the TODO principle.

\subsection{Flexibility vs adaptation.}

Food secking behaviour is a paradigm which has becn frequently used in studyirig various forms of (adaptive) behaviour specification (e.g. Booker (1982), Wilson 1985), Coderre (1988). Travis (1988)). It is instructive to compare the behaviour of an extremely simple TODO specification with that obtained by a sophisticated learning scheme. We did this for ANIMAT (Wilson 1985) (whose behaviour is governed by a classifier system (Holland 1986)) and compared it with SMARTY (with TODO based behaviour, which has the form "if you see food eat it; otherwise go straight") (Hogeweg 1989). We used the same environment, the same 'sensors', and the same experimental procedure in both cases(i.e. those reported by Wilson 1985). This experimental procedure admits of only half of the basic possibilities of TODO based entities: the environment is kept constant and therefore 'what there is to do' is not influenced by 'what is done'. This exercise makes clear that:

1. It is much easier to formulate SMARTY than it is to formulate ANIMAT and much less adhoc choices and tuning are necessary; 'hidden assumptions' play less crucial and unexpected roles (e.g. global or 'cgocentric' coding of direction).

2: SMARTY is a more efficient foodsceker than the best ANIMATS oblained.

3. When SMARTY is brought in a moderatcly different environment its behaviour degenerates less than that of ANIMAT. Also, in the long run, ANIMAT has a hard time to 'adapt' to the new environment, whereas SMARTY's behaviour is flexible enough to cope.

This example illustrates a conflict between adaptability and Mexibility. Information-rich representations of the environment (such as ANIMAT builds), which are reflected in local behavioural rules, impairs flexibility to cope with new environments. Coping with new environments is of course of paramount importance.' It is an open question how to cvolve flexihility: a variable environment is not enough (Rizki \& Conrad 1986).

\subsection{Emergent behaviour patterns.}

The previous example already suggests that informationpoor (nlexible) local behaviour specification leads to larger scale behaviour in which patterns in the environment are reflected, e.g. in a poor environmen SMARTY will move straight over long distances, whereas it will rigzag and remain in a small area in richer enviromments. In thes case this is not very striking because the lairgescalle behaviour is expressed in the same type of terms as the local bchaviour. However the macrobehaviour generated is not restricted to behaviour dimensions represented in the local rules.

Using again the foodgathering paradigm we studied such cmergent patterns in MIRRORworlds inhabited by CROWNs (Hogeweg \& Hesper 1990), CHIMPs (Hogeweg \& te Bockhorst 1990), and ORANGs (tc Boekhorst \& Hogeweg in prep). These worlds were shaped such that they resemble in some aspects the worlds of Crown of Thoms (a starfish from the coral reefs), chimpanzees and orang-utans respectively. In all cases individuals move to the nearest foodsource and eat it; moreover they may move towards each other. The resulting macrobchavioural patterns resemble closely those found in the corresponding REAL worlds:

1.The CROWNS are outbreak prone: i.e. they are inconspicuous and barcly influence their environments upto a certain density: slightly above that density they aggregate into large groups and entirely deplete the environment within a short time. Likewise Crown of Thorns depletes coral reefs, changing the entire ecosystem drastically for long periods (e.g. Endean \& Stablum, 1975; Moran, 1988). Sudden large increases of the population size is the most often assumed explanation. In contrast, in the CROWNworld the potentially large travelling spceds and the size of the CROWNs the usually large abundance of food (coral) and the local coral regrowth are the features responsible for this dramatic phenomenon.

2. In the CHIMP world we find travel groups of MALEs (who in search for mates approach every CHIMPlike entity in view), and lone FEMALEs. This group structure resembles closely the group structure (= social structure as defined by Rowell 1972) found in chimpanzees (Halparin 1979). The explanation of the occurrence of all male groups is usually sought in terms of defense against neighbouring groups. In the CHIMP world it is due to the food availability and the mutual CHIMPseeking behaviour.

3. In the ORANG world we lind travelling bands which form in small fruit trees, rather than in large ones. Travelling bands were found in orang utans in the season in which many small trees have fruits (Sugardjitu et al 1987), which was explained in terms of a compromise between a 'social urge' and avoidance of food competition. In the ORANG world it is a side effect of going to the nearest food source and cating until satisfied. This behaviour leads to travelling bands when food sources are depleted before the caters are satisfied, and when enough food sources are available. Reexamination of the data (tc Bockhorst \& Hogeweg in prep.) shows that this is indeed the way in which travelling bands are formed in the orang utans.

These examples show:

1. the large impact of the interplay hetween simple TODO rules and a specific envirnnment: it creales the environment in which the entitic's regularly find themselves: the depleted coral, the all matc cHINIt' gromps and the seasonal travelling 
ORANG bands.

2. How the patterns found in the TODO based artificial worlds reflect pattems found in REALworlds.

3. The apparently superfluous mechanisms and concepts used in the common explanation of these REALworld patterns.

The emergence of new environmental features by the interaction of TODO rules with the environment can be viewed as the emergence of new 'fitness' dimensions.

\subsection{Selfregulation of TODO based emergent patterns.}

The patterns discussed above, emerging from food seeking behaviour, are fairly robust to changes in the environmental conditions. This invariance is caused by a subtle interplay between changes in the environment and the resulting changes in the actions of the entities (see Hogeweg \& te Boekhorst 1990, Hogeweg \& Hesper 1990). However, the selfregulation is more clearly demonstrated in our older work on socioinformatic processes, i.e. TODO based interactions restricted to initially identical entities, which may, however, differentiate due to the interactions in which they participate. We studied socioinformatic processes in BUMBLE's (Hogeweg \& Hesper 1983,1985). BUMBLEs are entities who reflect the lifehistory patterns of bumble-bees: EGG, LARVA, PUPA, WORKER, QUEEN and DRONE stages are recognised. The TODO is triggered by the type (stage) of BUMBLE met: a LARVA is fed when a WORKER or QUEEN meets it etc. When adult BUMBLEs meet they engage in a so called DODOM interaction: with a probability proportional to the value of their respective DOM parameter one of them 'wins'. The outcome of the interaction results in an updating of the DOM parameters: The DOM parameter of the winning BUMBLE is increased with an amount inversely proportional to the probability of winning (and vice versa for the losing BUMBLE). Thus, a damped positive feedback of DOM values ensues. The DODOM interaction together with the maintenance interactions generate many features observed in life bumblebee nests: a stable differentiation between elite and common workers, a 'rebellion' of the worker at the end of the season, chaotic interactions afterwards, egglaying by only the elite workers.

Moreover, the emergent features are strongly selfregulating. For example, 'compensatory feeding' (Pendrel \& Plowright 1981) is observed after the removal of part of the worker force. In the BUMBLE world this is a simple consequence of the TODO structure: WORKERs meet more often a LARVA when there are fewer WORKERs, and therefore more often feed them; in bumblebees an inspection behaviour was thought to be responsible for this (adaptive) behaviour. Even more interesting, the timing of the worker rebellion is invariant for a large range of growth rates of the nest. This is clearly important because, according to ergonomic optimality theory (Oster \& Wilson 1978), the rebellion, and therewith the switch to the production of generative offspring, should occur at the end of the season. when there is just enough time left to rear the generative offspring. The invariance of this 'socially generated clock' works via the differentiation in common workers and elite workers. In fast growing nests this differentiation is less pronounced than in slow growing nests. Therefore a larger worker force is nceded to remove the QUEEN from the nest. Thus, in very. slow growing nests where ergonomic consideration do no longer apply, rebellion occurs earlier, as is indeced observed in REAL nests.

This example clearly demonstrates the tendency of TODO, 'do what there is to do', to generate automatically 'what should be done', i.e. to generate automatically 'adaptive behaviour'.

\subsection{TODO as basis of building, behaving BEINGS.}

The TODO based worlds, discussed so far, are all entirely symbolic, and are simulated on digital computers. Such a symbolic representation can both obscure and generate 'hard' problems (Pattee 1988, Brooks 1989) Profoundly new insights can therefore be obtained by actually building TODO based entities which live in the REALworld. Brooks (Brooks 1986,1989 Brooks \& Flynn 1989, Flynn \& Brooks 1990 ) is doing just this; his goal is to build autonomous BEINGS which can subsist in some real environment, pursueing their own 'goals'. Unlike previous robotics research, but similar to the pre-history of robotics, i.e. the Grey Walter turtles (Grey Walter 1953), and to the above discussed line of research. Brooks' BEINGs do not try to build a global model of the world in which they live, but instead use the actual (local) world as the best possible model of the world(Brooks 1989). They react on the input provided by their sensors in a TODO-like manner. However, a real environment is much more complex than the simulated environments discussed so far; bchaviour of a BEING is therefore governed by a number of simultaneously operating TODO like processes, each using their own observations of the local environment. They interact at the action level, rather than cooperating to build a more complete representation of the situation in which the BEING finds itself. Apart from state changes in the environment (which trigger other TODOs) Brooks uses for the interaction of the TODO like ACTORs his 'subsumption network' in which lower level processes (e.g. 'do not bump into objects') can inhibit higher level processes (e.g. 'explore').

An important design feature is the autonomy of the lower layers of the subsumption network: they are functionally independent of the presence of the higher layers. Moreover the higher levels are also in a sense 'autonomous' but in such a way that the world with which they interact is 'shaped' by the lower level processes (e.g. is shaped such that bumping into things does not occur). Modularity facilitates the design enourmously. Similar modularity apparently exists in biotic systems. However models of evolution usually do not exploit such modularity (see section 4). 


\subsection{Multiple TODO based worlds.}

The TODO principle, as discussed sofar, applics the TODO rules on the basis of information that is locally available in the environment in which the entitics dwell. Environments in which the entities can extract this local information and can survive on the basis of it, may be called 'benign' for the entities under consideration. In less benign environments, information obtained from the environment has to be supplemented, in order to survive, by information obtained from an internal model of the environment. Thus an internal model may serve as a 'crutch' for the inability to extract the information directly from the environment, and/or can serve as a means to obtain more global information. This can be seen by comparing the mechanisms for chemotaxis in bacteria and protozoa. The latter observe a chemotactic gradient directly by comparison of concentrations 'in front' and 'bchind'. The size of the former is too small to elevate this difference above noise levcl, and bacteria thereforc use an internal model, i.e. a memory of the concentration, to decide whether to go straight or to turn. (This suggests that good observers can afford to be bad theoreticians and viceversa! compare drowsing rod practicioners, whose successes are probably due to kecn local observation). It should be noted that in the case of the CHIMPS and ORANGs, discussed above, the observations used (i.c. the distance in which fruittrees were spotted) is probably beyond the obscrvational capacities of chimpanzecs and orangutans; instcad these animals are assumed by ficldworkers to have an internal model of the environment such that they 'know' whether a fruittree is present at such distances (Gondall 1986, Rijksen 1978).

The internal model needed in, in this sense, less 'benign environments might take a similar form as the TODO based worlds discused so far (c.g. Hogeweg \& Hesper 1986,1988). For examplc, Macs $(1989,1990)$ proposed as an alternative to the (hicrarchical) subsumption architecture of (Brooks (1986), a more 'hetcrarchical', but also low information content interaction between TODO-like ACTORS These ACTORs change each others environment in the sense of adding and deleting preconditions; morcover they pass activity levels toward cach other. Maes (1990) claims that by such an architecture the ACTORs collectively 'do the right thing', and continue to do so when new ACTURs are added to the network.

The similarity between such an ACTOR network and the TODO based models discussed above suggests a similarity with respect to emergence of macro-patterns (compare Hogeweg \& Hesper 1988). Thus, multiple levels of TODO based world can be envisioned as the basis for 'complcx' bchaviour.

\section{TODO based pattern processing.}

All examples reviewed above show that collections of simple TODO based entitics can be viewed as paltern processing devices. This pattern precessing can be viewed as pattern detection, pattern transformation or pattern generation whichever image one prefers. Although these are formally equivalent, they indicate different impacts of the pattern processing: we examine them in turn.

1. TODO as pattern detection. The robustness of the macropattems studied above for some differences in the environment can be interpreted in terms of pattern detection (classification): environments are classified into those generating the macropatterns discussed, and those gencrating some other pattern (compare e.g. CHIMPs vs ORANGs, although the TODOs are not exactly identical). The generated classes of the environment are 'labelled' with the macropattern generated, i.e. a qualitative difference is added to the initially present gradual differences.

2. TODO as pattern transformation (pattern association). Patterns in the environment are transformed via TODO rules into what is actually done. Equivalently one might say that patterns in the environment are associated with behaviour patterns. As we have seen the robustness of some macrobehavioural fealures is obtaincd by subtle and multiple changes in what is actually done. Thus, in viewing TOIO) based behaviour as a pattern transformation process, we do not focus on invariant macropatterns as is the case with pattern detcction, but focus on the variation in the macrobehaviour, which is caused by changes in the environmental conditions. Moreover, we focus on the change in representation of the patterns: from environmental patterns to patterns of behaviour. Such transformation potentially renders the patterns obscrvable for entities cquipped with other sensors (and other TODOS).

3. TODO as pattern generation. TODO based pattern processing tends to be selfenhancing. Environmental patterns which are transformed into behavioural patterns are doubly represented and therefore will be preferentially detected. This is in particular true for invariant features generated by a TODO in a set of environments: invariances are casily detectable. This selfenhancement, together with the transformation of scale of the patterns, and the introduction of new representations of the pattern makes that the pattern processing can often best be regarded as pattern generation: similar pattern processing may underlie processes primarily observed as pattem generation processes.

Examples discusscd in scction 2 show how TODO based pattern processing vicwed as pattern detcction, pattern transformation, or pattern generation, provides alternative explanations for pattems of bchaviour usually explainced in terms of evolutionary optimisation. TODO based paltem processing may, morcover, provide prepattems on which evolutionary processes operate (scction 4). 


\section{TODO as substrate for evolution.}

The emergent adaptive processes we have considered operate at a short (behaviour) time scale, whereas evolutionary adaptation is of course a long term process. Long term processes are usually considered to determine the short term behaviour. Coupling between 'genes' results in emergent patterns and rugged fitness landscapes. This constraints optimisation of an apriori chosen fitness criterion (expressed in terms of 'gene'states) (Kauffman 1986, 1988a,b). Thus, emergent pattems and evolution scem to oppose each other. In particular for autonomous evolutionary processes (i.e. evolutionary processes without a-priori specified fitness criterion) it is important to consider the influence of short term emergent patterns on long term evolutionary processes as well. This influence is multifacetted:

1. (Spatial) Patterning changes basic properties of evolutionary processes: e.g. spiral patterns emerging from hypercyclic interaction (Eigen \& Shuster 1979) in pnorly mixed media cause positive selection for 'altruistic' traits (hypercycles become resistent to parasites) (Boerlijst \& Hogeweg 1990)

2. The 'pattern detection' or 'pattern generation' properties of TODO rules (as described above) create (new) fitness dimensions, and (new) fitness criteria. As mentioned e.g. 'social' bchaviour becomes a fitness dimension when fecding interactions create (large) parties. At least as important: stable emergent patterns will lead to evolutionary loss of TODO's for situations never (seldom) encountered. This leads to the formation of the generated (detected) pattern becoming itself a fitness dimension.

3. The 'pattern transformation' properties of TODO rules (as described above) create prepatterns of regulatory 'adaptations' to produce the same emergent (generated/detected) patterns in different environments. Long term evolutionary processes may tend to strengthen such prepatterns (viz 2. above). Interestingly, Flynn \& Brooks (1989) note that layers added in the subsumption hierarchy to improve the walking (and climbing) abilities of an 'insect' with 6 separately controlled legs, sometimes only strengthens emergent behaviour already present in the system due to e.g. gravitation (e.g. "bend the leg on the ground when an other leg cannot reach a foothold").

Evolutionary (and learning) processes are most often studied in isolation: the device is supposed to be unstructured relative to the problem to be learned, i.e. it is initialised with as little prepattern as possible, i.c. the device is initialised 'randomly'. (viz. classical evolution theory (one trait, one de novo gene), genetic algorithm (random initial population, but see Huynen and Hogeweg (1989)), neural networks (random initial (intemal) state in pattern recognition; random or uniform initial weights in learning)).
Absence of 'prepatterns' is certainly not the case for any 'real world' evolutionary (or learning) process. It always starts in a 'viable' state in which its interactions with the environment can be scen as governed by TODO rules. At the very least gross physical TODO's are present (e.g. "if no support then fall down" (Flynn \& Brooks 1989)). As discussed, TODO rules lead to prepatterns which form a substrate for (autonomous) evolutionary processes.

Prepatterns are ignored when learning systems are studied in isolation. Such an approach can be called 'holistic' or 'reductionistic', opposites which are equivalent in the sense that interesting structures are left unanalysed. The pattern processing image of simple TODO rules sketched above, and the image of emerging patterns as substrate for evolution may lead to a modular description of evolving and evolved systems.

\section{References}

Ackley, D.H. Hinton, G.E. \& Sejnowski, T.J. (1985) A learning algorithm for Boltzmann machines. Cognitive Science 9: 147-169

teBoekhorst I.J.A. \& Hogeweg P. (1990) Tree size and travel bands in orang-utans: data-analysis suggested by a model (in prep).

Boerlijst, M.C. \& Hogeweg, P. (1990) Spiral wave structure in prebiotic evolution: Hypercycle stable against parasites. Physica D (submitted)

Booker, L.(1982) Intelligent Behaviour as an adaptation to a task environment PhD. Dissertation. The Univ. of Michigan

Brooks, R.A. (1986) A robust layered control system for a mobile robot. IEEE Journal of Robotics and Automation. RA-2: 14-23.

Brooks R.A. (1989) The whole Iguana. in: Brady M. (ed.) Robotics science MIT press pp: $432-458$

Brooks R.A. \& Flynn A.M. (1989b) A robot being. in NATO workshop on robotics and biological systems, Tuscany, Italy.

Coderre, B. (1988) Modelling Behaviour in Petworld. in: C. Lanton (ed.) Artificial Life. Addison Wesley Publ Co pp:407-420

Conrad M. \& Rizki M. (1989) The Artificial world approach to emergent evolution. Biosystems 23: 247-260

Eigen M. \& Shuster P. (1979) The Hypercycle, a principle of natural selforganisation Springer Verlag.

Endean R. \& Stablum, W. (1975) Population explosions of Acanthaster planci and associated destruction of the hard coral cover of reefs in the Great Barrier Reef, Australia. Environm Conserv 2: 247-259.

Flynn A.M. \& Brooks R.A. (1989) Building Robots: 


\section{expectitions and experientes}

Goldberg, D.E. (1989) Genctic Algorithms in search optimisation and machine learning. Addison Wesley Publ co.

Goodall 1. (1986) The Chimpanzees of Gombe. Belknap. Cambridge (MA)

Malperin S.D. (1979): Temporary association patterns in free ranging chimpanzees: an assessment of individual grouping preferences. In: Hamburg DA, McCown E.R. (cds) The great apes. Benjamin/Cummings, Menlo Park (Calif), pp: $491-499$.

Hogeweg P. Hesper B. (1983) The ontogeny of the interaction structure in Bumblebce colonies: a MIRROR model Behav, Ecol Sociobiol. 12: 271-283.

Hogewes P. \& Hesper B. (1985) Socioinformatic processes, a MIRROR modelling methodology. J Theor Biol. 113: 311-330.

Hogeweg P. \& Hesper B. (1986) Knowledge secking in variable structure models. in Modelling \& Simulation in the Artificial Intelligence Era (ed. Elzas, Oren \& Klir) Noxh Holland pp: $227-243$

Hogewreg P. \& Hesper B. (1987) Simulation Modelling Formaltsm: Heteranchical Systems. in Systems \& Control Encyclopedia (ed. M.G. Singh) Pergamon Press pp: $\mathbf{4 3 5 0 4} 4398$

Hogieweg P. (1988) MIRROR bcyond MIRROR, puddles of Life. Artificial Life, SFI Studies in the Seiences of Complexity (ed C. Langton) Addison Wesley PubL Co pp: 297-315

Hageweg. P. (1989) Simplicity and Complexity in MRRioR universes. Biosystems 23 231-246

Hogeweg P. \& Hesper B. (1990) Crowns crowding, an individual oriented model of the Acanthaster phenomenon. The acanthaster phenomenon. (ed. R. Bradbury) Springer Lecture Notes in Mathematical Biolozy (in press)

Hogeweg, P. \& te Bockhorst I.J.A. (1990) On the natural history of an artificial CHIMP world: group structure as a side-eftect of feoding behavior. (submitted)

Holland J.H.(1976) Adaptation in natural \& Artificial systems Univ, of Michigan Press, Ann Arbor

Halland, J.H. (1986) Escaping Brittleness: the possibitities of general purpose leaming algorithms applied to paralell rule-based systems. in: R.S. Michalski et al. (cds.) Machine Leaming Vol. 2 Morgan Kauffman, Los Alamos Ca.

Holland, J.H. (1990) Oral presentation at Artifial Life II Santa Fe.

Hopfield J.J. (1984) Neurons with graded response have collective computational properties like those of a two- state neurons PNAS 81: 3088-3092

Kauffman, S.A. \& Smith, R.G. (1986) Adapti automata based on darwinian selection. Physica D 22: 68- $\varepsilon$

Kauffman, S.A. (1989a) Adaptation in rugged fitne landscapes. in: D.L. Stein (ed.) Lectures in $t$ sciences of complexity Addison Wesley Publ. Co p $527-618$

Kauffman, S.A. (1989b) Principles of adaptation complex systems. in: D.L. Stein (ed.) Lectures in th sciences of complexity Addison Wesley Publ. Co p $619-712$

Maes, P. (1989) The dynamics of action selection. IJC t 1989.

Maes, P. (1990) How to do the right thing.

Moran, P.J. (1988) The Acanthaster Phenomenol Australian Institute of Marine Science Monograph series n 7. Townsville, Australia.

Oster G.F.\& Wilson E.O.(1978) Caste \& Ecology in th. social insects. Princeton Univ. Press Princeton.

Packard, N. (1989) Evolving bugs in a simulate ecosystcm. in:Langton C. (ed.) Artificial Life. Addiso Wesley . pp:141-156.

Pattec H.H. (1988) Simulations. Realisations an thcories of life. in: C. Langton (ed.) Artificial Lift Addison Wesley, pp:49-63

Pendrel, A.A. \& Plowright, R.C. (1981) Larval feedin by adult bumble bee workers (hymenoptera: Apidae Behav. Ecol Sociobiol 8: 71-76

Rizki M.M. \& Conrad M. (1986) Computing the theor of evolution. Physica D 22: 83-99.

Rowell T.E. (1972) The social behaviour of monkeys Pinguin books Baltimore

Rijksen, H.D. (1978) A Field Study ' on Sumatras Orang-utans (Pongo pygmaeus abelii, Lesson 1982) Ecology, behaviour \& conservation. H. Veenman \& Zonen, Wageningen (Netherlands).

Simon, H. (1969) The Sciences of the Artificial, MI] press.

Sugardjito J, teBoekhorst I.J.A.\& van Hooff J.A.R.A.M (1987) Ecological constraints on the grouping of wilc orang-utans (Pongo pygmacus) in the Gunung Leuses National Park Sumatera, Indonesia. Int.J. Primatol 8:17-41.

Travers, M. (1988) Animal construction kits. in: C. Langton (ed.) Artificial Life. Addison Wesley pp. 421-442 Walter, W.G. (1953) The Living Brain. Norton, New York. Wilson, S.W. (1985) Knowledge growth in an artificial animal in: J.J. Grefenstette (ed.) Genetic Algorithms. (Carnegie Mellon. Piusburg PA). 